\title{
On Abstract Numerical Integrations
}

\author{
John J. Sopka* \\ National Bureau of Standards, Boulder, Colo.
}

(March 10, 1966)

\begin{abstract}
Let $X$ be a space of functions, say $X \subset C(K), K$ locally compact Hausdorff, let $l \epsilon X^{*}$ be an integral on $X$ and let $M^{*} \subset X^{*}$ be a given subspace of "simple" functionals, then it is desired to obtain an $\bar{l} \epsilon M^{*}$ for given $n, \bar{l} \epsilon M_{n}^{*} \subset M^{*} ; M_{n}^{*}$ being a suitable $n$ dimensional subspace determined so that: $l-\bar{l}$ annihilates a given finite dimensional subspace $X_{1} \subset X$. In this general context, the abstract analysis of numerical integration is developed and certain specific applications are made.
\end{abstract}

Key Words: Numerical integrations, abstract Gaussian quadrature methods.

\section{Introduction}

In the development of numerical integration formulas, one is given a function space $X$, say $X \subset C(K)$, where $K$ is some locally compact Hausdorff space, and an integral $l \epsilon X^{*}$. It is then desired to find an $\bar{l} \epsilon M^{*} \subset X^{*}$, where $M^{*}$ is a subspace generated by a family of "simple" integrals $\left\{x_{\alpha}^{*}\right\}$ such that $\bar{l}$ can be said to approximate $l$ in some given sense.

In ordinary quadrature, the family $\left\{x_{\alpha}^{*}\right\}$ are given by $x_{\alpha}^{*} f=f\left(x_{\alpha}\right) \vee f \in C(K)$ where $x_{\alpha} \in K$. An element $\bar{l} \epsilon M^{*}$ is said to approximate the given integral $l$ if $1-\bar{l} \epsilon X_{\omega}^{\perp}$ where $X_{\omega}^{\perp}$ is the subspace in $X^{*}$ of all annihillators of a given subspace $X_{\omega} \subset X$.

The following paper summarizes the essential mathematical structure of numerical integration methods with the purpose of extending systematically the class of such methods.

\section{General Analysis}

Let $X \subset C(K)$ and let $\mathrm{X}_{0} \subset X$ be a subspace $\ni \operatorname{dim}$ $X_{0}=n$ and $X_{0}$ is generated by a given set of $n$ "basis" functions $\left\{f_{k}\right\}$. In $X^{*}$ take $M^{*}$ a subspace generated by a given family $\left\{x_{\alpha}^{*}\right\}$ of linearly independent functionals with the properties: $M^{*}$ is total over $X_{0}$ and $\left\{x_{\alpha}^{*}\right\} \cap X_{0}^{\perp}$ is void where $X_{0}^{\perp}=\left\{l ; l \epsilon X^{*}, l f=0, f \epsilon X_{0}\right\}$. If $M_{n}^{*} \subset M^{*}$ designates a subspace generated by $n$ of the $x_{\alpha}^{*}$, say $\left\{x_{\alpha i}\right\} i=1 \ldots n$ so that $\operatorname{dim} M_{n}^{*}=n$, then one has $X^{*}=X_{0}^{\perp}+M_{n}^{*}$ since the deficiency of $X_{0}^{\perp}=\operatorname{dim}$ $X_{0}=n$. Also $M_{n}^{*}$ is total over $X_{0}$ as is readily seen.

If $l \epsilon X^{*}$ is a given integral, then $\exists \bar{l} \epsilon M_{n}^{*}{ }^{*} l=\bar{l}+l_{0}^{\perp}$ where $l_{0}^{\perp} \epsilon X_{0}^{\perp}$ and $\exists$ such an $\vec{l}$ for each subspace $M_{n}^{*}$ as defined above. For any such given subspace $M_{n}^{*}$ $\exists l_{i} \quad \epsilon M_{n}^{*} \quad i=1 \ldots n_{\ni} l_{i} f_{k}=\delta_{i k} \quad i, k=1 \ldots n \quad \delta_{i k}=1$ $i=k, \delta_{i k}=0 i \neq k$. This follows from $M_{n}^{*}$ being total over $X_{0}$.

*Consultant-Mathematics, National Bureau of Standards, Boulder, Colo.
Thus one can write: $l=\sum_{i} l\left(f_{i}\right) l_{i}+l_{0}^{\perp}, l_{0}^{\perp} \epsilon X_{0}^{\perp}$ and $l_{i}=\sum_{j} \gamma_{i j} x_{j}^{*}$ where $x_{j}^{*}=x_{\alpha, j}^{*}$.

Then

$$
\begin{gathered}
l=\sum_{i} \sum_{j}\left(l f_{i}\right) \gamma_{i j} x_{j}^{*}+l \frac{\perp}{0} \\
\bar{l}=l-l \frac{\perp}{0}=\sum_{j}\left(\sum_{i} l f_{i} \gamma_{i j}\right) x_{j}^{*} .
\end{gathered}
$$

The functional $\bar{l}$ therefore depends on the selected set $\left\{x_{\alpha i}^{*}\right\}$, that is on $M_{n}^{*}$, on the $l f_{i}$ and on the coefficients $\gamma_{i j} i, j=1 \ldots n$.

The relations $\sum_{j} \gamma_{i j} x_{j}^{*} f_{k}=\delta_{i k} i, k=1 \ldots n$ can be put in matrix form: $\Gamma \Gamma_{0}=I$ where $I$ is the identity matrix, $\Gamma=\left(\gamma_{i j}\right) \Gamma_{0}=\left(x_{j}^{*} f_{k}\right)$. Now $\Gamma_{0}$ is nonsingular for any given $M_{n}^{*}$ since $M_{n}^{*}$ total over $X_{0}$ implies that $\sum_{k=1}^{n} \tau_{k} x_{j}^{*} f_{k}=0 j=1 \ldots n$ is possible only when $\tau_{k}=0$ $k=1 \ldots n$. Hence the matrix $\Gamma=\Gamma_{0}^{-1}$ is determined once $\Gamma_{0}$ is known.

If the $\left\{f_{k}\right\}$ are such that the $l f_{k}$ are readily evaluated then the problem of determining $\bar{l}$ is that of specifying $M_{n}^{*}$ or a corresponding basis $\left\{x_{\alpha i}^{*}\right\}$. Under the conditions imposed thus far with $\operatorname{dim} X_{0}=\operatorname{dim} M_{n}^{*}=n$ $\bar{l}$ will not in general be unique without additional conditions on $X$ and/or the $M_{n}^{*}$.

\section{Specific Cases}

In certain quadrature procedures, such as the Newton-Cotes formulas, $M_{n}^{*}$ is specified a priori by choosing the $x_{\alpha i}^{*}$. The subspace $X_{0}$ in these cases is such that the $l f_{i}$ are easily calculated and thus $\bar{l}$ 
is determined. For instance, the Parabolic Rule has $l=\int_{x_{1}}^{x_{3}} d x$, where $X_{o}$ is the space over the Reals generated by the $f_{k}=x^{k-1}, k=1,2,3$ and $x_{\alpha i}^{*} f=f\left(x_{i}\right)$ for given real numbers $x_{1}, x_{2}=\frac{1}{2}\left(x_{1}+x_{3}\right), x_{3}$.

In Gaussian quadrature methods, the following additional conditions limit the choice of $M_{n}^{*}$. Consider a subspace $X_{1} \subset X$, where $X_{1} \supset X_{0}$ and $X_{1}$ has generators $\left\{f_{k}^{\prime}\right\}, k=1 \ldots p, p>n, f_{k}^{\prime}=f_{k}$ for $k=1 \ldots n$. Now if $T$ is a linear transformation on $X \ni f_{k}^{\prime}=T f_{k-1}^{\prime}$ $=T^{k-1} f_{1}^{\prime}$. Then $T$ induces a linear transformation $Q$ on $X^{*}$ given by $Q l f=l f=l T f, \quad \forall l \epsilon X^{*}, \quad f \in X$. Consequently $x_{j}^{*} f_{k}^{\prime}=\left(Q x_{j}^{*}\right) f_{k-1}^{\prime}=\left(Q^{k-1} x^{*}\right) f_{1}^{\prime}$ and one can write:

$$
\bar{l} f_{k}=\sum_{j}\left(\sum_{i} l f_{i} \gamma_{i j}\right) Q^{k-1} x_{j}^{*} f_{1} \text { for } k=1 \ldots p .
$$

Since the matrix $\Gamma_{0}=\left(x_{j}^{*} f_{k}\right)=\left(Q^{k-1} x_{j}^{*} f_{1}\right)$ for $j$, $k=1 \ldots n$ is nonsingular, the $n$-tuple $\left(Q^{n} x_{j}^{*} f_{1}\right)$ $j=1 . . . n$ corresponding to

$$
\bar{l} f_{n+1}=\sum_{j}\left(\sum_{i} l f_{i} \gamma_{i j}\right) Q^{n} x_{j}^{*} f_{1}
$$

is linearly dependent on the rows of $\Gamma_{0}$, i.e.

$$
Q^{n} x_{j}^{*} f_{1}=\sum_{k=1}^{n} \beta_{k} Q^{k-1} x_{j}^{*} f_{1} \quad j=1 \ldots n .
$$

This gives the relationship

$$
\bar{l} f_{n+1}^{\prime}=\sum_{k=1}^{n} \beta_{k} \bar{l} f_{k}^{\prime} .
$$

Now, if for $M_{n \perp}^{*}=\left\{f ; f \in X_{1}, x_{j}^{*} f=0, j=1 \ldots n\right\}$ one also assumes: $T M_{n \perp}^{*} \subset M_{n \perp}^{*}$ that is, $x_{j}^{*} f=0$ implies $x_{j}^{*} T f=0=Q x_{j}^{*} f$ for $j=1 \ldots n$ then this condition, plus the statement:

$$
\left(Q^{n}-\sum_{1=1}^{n} \beta_{k} Q^{k-1}\right) x_{j}^{*} f_{1}^{\prime}=0 \quad j=1 \ldots n
$$

will imply that

$$
\left(Q^{n+q}-\sum_{k=1}^{n} \beta_{k} Q^{k+q-1}\right) x_{j}^{*} f_{1}^{\prime}=0 \quad j=1 \ldots n, \forall q
$$

Taking $q=0,1 \ldots n-1$ one gets the $n$ equations

$$
\bar{l} f_{n+1+q}^{\prime}=\sum_{k=1}^{n} \beta_{k} \bar{l} f_{k+q}^{\prime}
$$

In Gaussian quadrature, the stronger condition $l-\bar{l} \epsilon X_{1}^{\perp} \subset X_{0}^{\perp}$ is imposed. Thus, if $l-\bar{l} \epsilon X_{1}^{\perp}$, if the $f_{k+q}^{\prime}$ are such that the $l f_{k+q}^{\prime}$ are readily obtained and if the matrix $L=\left(l f_{k+q}^{\prime}\right), k=1 \ldots n, q=0 \ldots n-1$ is nonsingular then the $\beta_{k}$ can be obtained uniquely.
Given the $\beta_{k}$, solution for the $x_{j}^{*} f_{1}^{\prime} j=1$. . . n follows from the relations (5) when they are solvable. Determination of the functionals $x_{j}^{*} j=1 \ldots n$ proceeds from the values of the $x_{j}^{*} f_{1}$ when the $x_{j}^{*}$ are sufficiently simple, for example, a one parameter family like those in the examples below.

Now, $L=\left(l f_{k+q}^{\prime}\right)$ is nonsingular if and only if scalars

$$
\begin{aligned}
\epsilon_{k} \neq 0, k=1 \ldots n \ni l\left(\sum_{k=1}^{n} \epsilon_{k} f_{k+q}\right)= & \\
& \\
q & =0 \ldots n-1 .
\end{aligned}
$$

Since these last relations can be written as:

$$
l\left(\sum_{k=1}^{n} \epsilon_{k} T^{k-1+q} f_{1}\right)=l\left(T^{q}\left(\sum_{k=1}^{n} \epsilon_{k} T^{k-1}\right) f_{1}\right)=0
$$

one has, in this general context, an orthogonality condition stating that no $n-1$ degree "polynomial" in $T$ is orthogonal in this sense to all $n-1$ degree polynomials in $T$. The $n$-equations (7) can also be written as:

$$
l\left(T^{q}\left(T^{n}-\sum_{k=1}^{n} \beta_{k} T^{k-1}\right) f_{1}\right)=0 \quad q=0 \ldots n-1
$$

which states that the $n$ degree polynomial $T^{n}-$ $\sum_{k=1}^{n} \beta_{k} T^{k-1}$ is orthogonal, in this sense, to all $n-1$ degree polynomials in $T$.

Thus if $X_{0}, X_{1}, M_{n}^{*}$ satisfy the conditions imposed, it was seen in the general section above that $\exists \bar{l} \epsilon M_{n}^{*} \ni$ $l-\bar{l} \epsilon X_{0}^{\perp}$. The preceding discussion shows that if $l-\bar{l} \epsilon X_{1}^{\perp}$ then (9) follows. Conversely, if $\exists \beta_{k} \ni$ (9) holds one shows directly $l-l \epsilon X_{1}^{\perp}$ using (5) and (6). Consequently one has the following statement analogous to the ordinary Gaussian quadrature theorem [1] which is usually stated in terms of classical orthogonal polynomials: If $X_{0}, X_{1}, M_{n}^{*}$ satisfy all the conditions imposed above then $\exists \bar{l} \epsilon M_{n}^{*}{ }_{\ni} l-\bar{l}_{\epsilon} X_{1}^{\perp}$ if and only if $\exists$ an $n$ degree "polynomial" $T^{n}-\sum_{k=1}^{n} \beta_{k} T^{k-1}$ orthogonal as in (9) to all polynomials in $T$ of degree $<n$.

\section{Applications}

In ordinary Gaussian quadrature, the above specializes to the case where $X_{0}=\left\{x^{i-1} ; i=1 \ldots n\right\}$, $X_{1}=\left\{x^{i-1} ; i=1 \ldots 2 n\right\}$, and the $\left\{x_{\alpha}^{*}\right\}$ are defined by $x_{\alpha}^{*} f=f\left(x_{\alpha}\right)$. Then the other Gaussian conditions are seen to hold when $T$ is $\ni(T f)(x)=f(x) x$.

An interesting nonclassical application is to $l=\int_{0}^{1} d x$ on the space $X=C_{0}(K)=\{f ; f \epsilon C(K), K=[0,1], f(0)=f(1)$ $=0\}$. The natural basis functions here are $\sin \pi k x$ or the equivalent functions $f_{k}(x)=\sin \pi x(\cos \pi x)^{k-1}$. 
Take $X_{0}, X_{1}$, with this basis and $x_{\alpha}^{*}$ defined by $x_{\alpha}^{*} f=f\left(x_{\alpha}\right), \quad x_{\alpha} \in[0,1]$. Take $T \ni T f(x)=f(x) \cos \pi x$ then $T M_{n \perp}^{*} \subset M_{n \perp}^{*}$ and one gets:

$$
\bar{l}=0.39\left(x_{1}^{*}+x_{2}^{*}\right)
$$$$
\bar{l}=0.205\left(x_{1}^{*}+x_{3}^{*}\right)+0.247\left(x_{2}^{*}+x_{4}^{*}\right)
$$$$
\text { for } n=2
$$$$
\bar{l}=0.18502\left(x_{1}^{*}+x_{2}^{*}\right)+0.12306\left(x_{3}^{*}+x_{4}^{*}\right) \quad \text { for } n=6 \text {. }
$$$$
+0.16882\left(x_{5}^{*}+x_{6}^{*}\right)
$$

Another application is to $l=\int_{0}^{\infty} k(x) d x$ for some weight function $k(x)$ e.g., $k(x)=e^{-x^{2}}$. Here $K=[0, \infty]$ $X=\left\{f ; f \epsilon C(K)\right.$, lf exists, $\int_{0}^{x} f(t) d t$ exists and is easily obtained for arbitrary $x \in K\}$.

Take $f_{k}^{\prime}(x)=k x^{k-1}$, let $x_{j}^{*} f=k\left(\xi_{j}\right) \int_{x_{j-1}}^{x_{j}} f(x) d x$. For $x_{0}$ $=0 \quad x_{j-1}<\xi_{j}<x_{j}$ and take $T$ given by $T f(x)=x f(x)$ $+\int_{0}^{x} f(t) d t$ then $T M_{n \perp}^{*} \subset M_{n_{\perp}}^{*}$ and one gets: $\bar{l}=0.922 \int_{x_{0}}^{x_{1}} d x+0.195 \int_{x_{1}}^{x_{2}} d x$ for $n=2$.

where $x_{0}=0, x_{1}=0.754, x_{2}=1.734$

$$
\begin{aligned}
\bar{l}=0.9798 \int_{x_{0}}^{x_{1}} d x+0.6188 \int_{x_{1}}^{x_{2}} d x+0.1386 \int_{x_{2}}^{x_{3}} d x & \\
+ & 0.0051 \int_{x_{3}}^{x_{4}} d x
\end{aligned}
$$

for $n=4$ where $x_{0}=0, x_{1}=0.4239 \quad x_{2}=1.014$

$$
x_{3}=1.792 \quad x_{4}=2.640
$$

$\bar{l}=0.99200 \int_{x_{0}}^{x_{1}} d x+0.81425 \int_{x_{1}}^{x_{2}} d x+0.41032 \int_{x_{2}}^{x_{3}} d x$

$$
+0.09445 \int_{x_{3}}^{x_{4}} d x
$$

$$
+0.00702 \int_{x_{4}}^{x_{5}} d x+0.00008 \int_{x_{5}}^{x_{6}} d x
$$

for $n=6$

where $x_{0}=0 \quad x_{1}=0.27779 \quad x_{2}=0.68991$

$$
\begin{array}{ll}
x_{3}=1.2090 & x_{4}=1.8138 \\
x_{6}=3.3556 .
\end{array}
$$

Greater detail on the development of such formulas and their utility will be given in a subsequent paper.

\section{References}

[1] Hildebrand, F. B., Introduction to Numerical Analysis, p. 319 (Mc-Graw-Hill Book Co., Inc., New York, N.Y. 1956).

[2] Ezrohi. I. A., General Forms of the Remainder Terms of Linear Formulas in Multidimensional Approximate Analysis I and II. Math Sbornik 38 (80) p. 389; Math Rev. 18,33. Math Sbornik 43 (85) p. 9; Math Rev. 19, 1199.

[3] Hammer, P. C., Numerical Evaluation of Multiple Integrals on Numerical Approximation, p. 99, ed. R. E. Langer, University of Wisconsin (1959).

[4] Remez, E. I., On Certain Classes of Linear Functionals in the Spaces $\mathrm{C}_{\mathrm{p}}$ and on the Remainder Term in Formulas of Approximate Analysis I and II. Trudy In-Ta Matem Akad, Nauk, Ukr. S.S.R.3, p. 2l. Trudy In-Ta Matem, Akad, Nauk, Ukr. S.S.R.4, p. 47, Math Rev 2, 195.

[5] Sard, A., Function spaces and approximation, Amer. Math. Soc. Numerical Analysis Proc. Symp. Appl. Math 6, 177.

[6] Day, M. M., Normed Linear Spaces (Springer, Berlin, 1958).

(Paper 70B-173) 